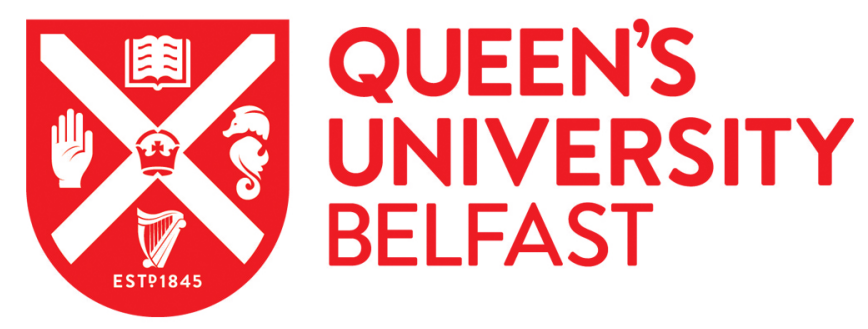

\title{
A curvilinear approach to the kinetic analysis of linoleate peroxidation in aqueous liposomes by 2,2'azobis(2-amidoinopropane) dihydrochloride
}

McPherson, P. A. C., Bole, A., Cruz, KA., Young, I., \& McEneny, J. (2012). A curvilinear approach to the kinetic analysis of linoleate peroxidation in aqueous liposomes by 2,2'azobis(2-amidoinopropane) dihydrochloride. Chemistry and Physics of Lipids, 165(6), 682-688. https://doi.org/10.1016/j.chemphyslip.2012.07.004

Published in:

Chemistry and Physics of Lipids

Document Version:

Peer reviewed version

Queen's University Belfast - Research Portal:

Link to publication record in Queen's University Belfast Research Portal

\begin{abstract}
Publisher rights
This is the author's version of a work that was accepted for publication in Chemistry and Physics of Lipids. Changes resulting from the publishing process, such as peer review, editing, corrections, structural formatting, and other quality control mechanisms may not be reflected in this document. Changes may have been made to this work since it was submitted for publication. A definitive version was subsequently published in Chemistry and Physics of Lipids, VOL 165, ISSUE 6, 09/2012
\end{abstract}

\section{General rights}

Copyright for the publications made accessible via the Queen's University Belfast Research Portal is retained by the author(s) and / or other copyright owners and it is a condition of accessing these publications that users recognise and abide by the legal requirements associated with these rights.

\section{Take down policy}

The Research Portal is Queen's institutional repository that provides access to Queen's research output. Every effort has been made to ensure that content in the Research Portal does not infringe any person's rights, or applicable UK laws. If you discover content in the Research Portal that you believe breaches copyright or violates any law, please contact openaccess@qub.ac.uk. 


\section{Accepted Manuscript}

Title: A curvilinear approach to the kinetic analysis of linoleate peroxidation in aqueous liposomes by 2,2'azobis(2-amidoinopropane) dihydrochloride

Authors: Peter A.C. McPherson, Amanda Bole, Kenneth A. Cruz, Ian S. Young, Jane McEneny

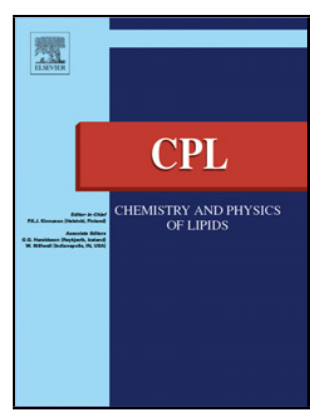

PII: S0009-3084(12)00083-7

DOI:

Reference: doi:10.1016/j.chemphyslip.2012.07.004

To appear in: $\quad$ Chemistry and Physics of Lipids

Received date: $\quad$ 7-4-2012

Revised date: 27-6-2012

Accepted date: $\quad$ 16-7-2012

Please cite this article as: McPherson, P.A.C., Bole, A., Cruz, K.A., Young, I.S., McEneny, J., A curvilinear approach to the kinetic analysis of linoleate peroxidation in aqueous liposomes by 2,2'azobis(2-amidoinopropane) dihydrochloride, Chemistry and Physics of Lipids (2010), doi:10.1016/j.chemphyslip.2012.07.004

This is a PDF file of an unedited manuscript that has been accepted for publication. As a service to our customers we are providing this early version of the manuscript. The manuscript will undergo copyediting, typesetting, and review of the resulting proof before it is published in its final form. Please note that during the production process errors may be discovered which could affect the content, and all legal disclaimers that apply to the journal pertain. 


\section{A curvilinear approach to the kinetic analysis of linoleate peroxidation in aqueous} liposomes by 2,2'azobis(2-amidoinopropane) dihydrochloride

Peter A. C. McPherson ${ }^{\mathrm{a}}$, Amanda Bole ${ }^{\mathrm{a}, \mathrm{b}}$, Kenneth A. Cruz ${ }^{\mathrm{a}, \mathrm{b}}$, lan S. Young ${ }^{\mathrm{c}}$ and Jane McEneny ${ }^{\mathrm{C}}$

${ }^{a}$ School of Applied Science (Chemistry), Belfast Metropolitan College, Belfast, UK

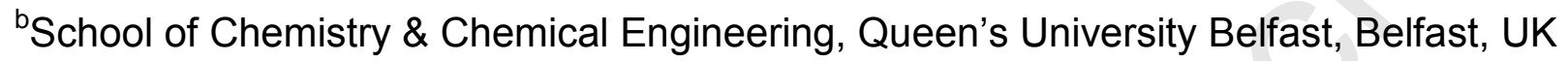

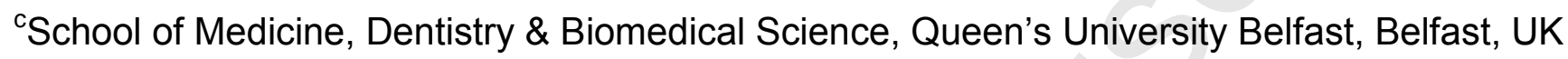

Corresponding author:

Peter A C McPherson, School of Applied Science (Chemistry), Belfast Metropolitan College, Titanic Quarter Campus, Queen's Road, Belfast, BT3 9DT, United Kindgom.

Email pmcpherson@belfastmet.ac.uk

Tel: + 442890265000

Fax: + 442890265001

Abbreviations:

$\tau$, lag time; $v_{\max }$, maximum rate; $\lambda_{\max }$, maximum wavelength; $\varepsilon_{\max }$, molar absorption coefficient for the specified maximum wavelength; AAPH, 2,2'azobis(2-amidoinopropane) dihydrochloride; $A_{\max }$, maximum absorbance; $\mathrm{BHT}$, butylated hydroxytoluene; E,E-13-HPODE, E,E-13-hydroperoxy-9,11octadecadienoic acid; E,E-9-HPODE, E,E-9-hydroperoxy-10,12octadienoic acid; exp, Euler's number; GRG2, generalised gradient reduction algorithm; HDL, high density lipoprotein; LDL, low density lipoprotein; RSS, residual sum of squares; $t_{\max }$, time at maximum rate; VLDL, very low density lipoprotein.

Key words:

Conjugated dienes; free radicals; Richards' equation; lag time; lipid hydroperoxides; Rice-Herzfeld mechanism; sigmoid curves 
1 Abstract

Lipid peroxidation is a common feature of many chemical and biological processes, and is governed by a complex kinetic scheme. A fundamental stage in kinetic investigations of lipid peroxidation is the accurate determination of the rate of peroxidation, which in many instances is heavily reliant on the method of finite differences. Such numerical approximations of the first derivative are commonly employed in commercially-available software, despite suffering from considerable inaccuracy due to rounding and truncation errors. As a simple solution to this, we applied three empirical sigmoid functions (viz. the Prout-Tompkins, Richards \& Gompertz functions) to data obtained from the AAPH-mediated peroxidation of aqueous linoleate liposomes in the presence of increasing concentrations of Trolox, evaluating the curve fitting parameters using the widely-available Microsoft Excel Solver add-in. We have demonstrated that the five-parameter Richards' function provides an excellent model for this peroxidation, and when applied to the determination of fundamental rate constants, produces results in keeping with those available in the literature. Overall, we present a series of equations, derived from the Richards' function, which enables direct evaluation of the kinetic measures of peroxidation. This procedure has applicability not only to investigations of lipid peroxidation, but to any system exhibiting sigmoid kinetics. 
1

2

\section{Introduction}

Peroxidation of unsaturated fatty acids is a common feature of many chemical and biological processes, and has been shown to proceed via a well-defined free radical chain reaction, involving the formation of conjugated dienes (Sevanian \& Hochstein, 1985). Due to their continuous $\pi$-bonding system, conjugated dienes absorb electromagnetic radiation in the $230-235 \mathrm{~nm}(\mathrm{UV})$ regions $\left(\lambda_{\max } 234 \mathrm{~nm} ; \varepsilon_{\max } 2.95 \times 10^{4} \mathrm{M}^{-1} \mathrm{~cm}^{-1}\right)$ (Antolovich et al. 2002) and when examined over these wavelengths, a time-dependent increase in UV absorbance is observed, which reflects the classical free radical sequence of initiation, propagation and termination (Schneider et al., 1998). Such free radical processes are subject to autocatalysis, and accordingly, plots of absorbance vs. time have an overall sigmoid appearance, with distinct regions of the sigmoid curve corresponding to a particular stage of the peroxidation chain reaction (Giseg \& Esterbauer, 1994; Raveh et al., 2000).

Initiation is a relatively slow process, in which allylic hydrogen atoms are abstracted from cis-cis pentadiene centres due to low bond dissociation energies (Porter et al., 1994), and is represented as a lag in UV absorbance, which may be quantitatively measured by the lag time $\left(t_{\text {lag }}\right)$ of the reaction (Cadenas \& Sies, 1998). The propagation phase, which involves the rapid production of conjugated dienes, is characterised by an exponential increase in UV absorbance, and can be assessed in two main ways: the maximum rate of oxidation $\left(v_{\max }\right)$, and the time at which maximum rate was achieved $\left(t_{\max }\right)$ (Pinchuk \& Lichtenberg, 2002). Termination of the free radical chain reaction may arise from biradical quenching, and produces (inter alia) lipid hydroperoxides, which decompose to a variety of aldehydes, ketones and hydrocarbons. This manifests as an asymptote to the $x$-axis, which gradually declines as the decomposition reactions advance (Porter et al., 1981).

5

A widely applied strategy for evaluating lipid peroxidation is the continuous monitoring of conjugated diene production at $\lambda_{\max } 234 \mathrm{~nm}$ (Esterbauer et al. 1989). However, in many 
1 routine investigations, data from such a technique is processed using finite differences (an

2 approximation of the first derivative), which is then used to determine $v_{\max }$ and the rate

3 constants for the reaction, as well as for the graphical determination of lag time (Fig. 1).

4 Such approaches are useful and relatively straightforward, but approximating first derivatives

5 numerically is subject to a range of errors, particularly rounding and truncation errors and

6 where possible analytical expressions should be used (Morton \& Mayer, 2005). Since a

7 variety of autocatalytic reactions have been successfully modelled using sigmoid functions

8 (Herney-Ramireza et al., 2011), a similar approach may provide a more precise means of

9 determining the kinetic constants of lipid peroxidation.

10

11

12

13

Analysis of sigmoid curves can be approximated by composite methods (Leith et al., 1996), but it is often more appropriate to use a curvilinear approach to ensure smooth transitions from one stage to the next. Such curvilinear equations are referred to as sigmoid functions, first proposed by Verhulst (Verhulst, 1839), and later used by Prout and Tompkins to describe the decomposition of potassium manganate(VII) (Brown \& Glass, 1999) The Prout-Tompkins equation has the integrated form:

$$
y=\frac{a}{1+\exp [-b(x-c)]}
$$

in which the parameter $a$ is the upper asymptote, $b$ is a curvature constant and $c$ is the point of inflection at which the curvature changes from convex to concave, or vice versa. Despite the considerable utility of such four-parameter sigmoid functions, the inherent symmetry around the parameter $c$ makes them unsuitable for more complex kinetic profiles. Richards (Richards, 1959) introduced an additional curvature constant, $d$, to account for asymmetry:

$$
y=a[1+b \exp (-c x)]^{1 /(1-d)}
$$


1 The asymmetry described by Richards' equation is at the expense of an additional

2 parameter, which in terms of physicochemical or biological processes, has no actual

3 meaning, and is therefore difficult to estimate. An alternative approach, in which the

4 Richards' parameter $d \rightarrow 0$, was described by Gompertz (Gompertz, 1825) and contains only

5 three parameters:

6

$$
y=a \exp \{-\exp [(x-c \cdot b)]\}
$$

A variety of sigmoid functions have been successfully applied to models of bacterial growth (Dalgaard \& Koutsoumanis, 2001; Simon \& Karim, 2001), the baroreceptor reflex (McDowall \& Dampney, 2006), pharmacological concentration-effect curves (Giraldo et al., 2002) and the crystallization of fats (Foubert et al., 2003). However, to the best of our knowledge, this approach has not been applied to lipid peroxidation. A conceptually similar method was reported by Molinari and co-workers in which splines were used to model peroxidation of low density lipoprotein (Molinari et al., 2002). This method was particularly effective in evaluating the peroxidation of lipids, although the mathematics of the process is likely to be less familiar to many researchers.

Evaluation of the kinetics of lipid peroxidation is a well-established field, and the rate constants for many common substrate-oxidant systems have been well-characterised (Antunes et al., 1996). To achieve this, kinetic data can be evaluated using finite differences, or by dedicated curve fitting software. Although this latter technique is undoubtedly very accurate, we sought to model lipid peroxidation using empirical sigmoid functions, establishing the curve fitting parameters using the Microsoft Excel Solver. This approach does not require any specialised software, or programming skills, and provides a fast, accurate means of evaluating kinetic data. In so doing, we propose an alternative 
1 definition of lag time, as the time at which the change in the rate of peroxidation is maximal

2 (the maximum of the second derivative).

3

\section{Materials \& Methods}

\subsection{Materials}

All chemicals were of at least analytical grade and used as supplied by Sigma-Aldrich (Poole, UK) unless otherwise stated. All aqueous solutions were prepared using Milli-Q double-deionised water (resistance $>18 \mathrm{~m} \Omega / \mathrm{cm}^{2}$ ) (Millipore, Bedford, MA, USA) stored over Chelex-100 resin. All absorbance measurements were made on a Shimadzu UV-visible 240 spectrophotometer (Antwerp, Belgium) attached to a PC for data acquisition.

\subsection{Substrate Preparation}

Linoleate liposomes were prepared weekly using a standard method (Surrey, 1964) with minor modification. In brief, $250 \mu \mathrm{L}$ of neat linoleic acid (3.2 M) were added drop wise, with stirring, to $5 \mathrm{~mL}$ of borate buffer $(0.05 \mathrm{M}, \mathrm{pH}$ 9) containing $5 \%$ Tween-20 and $0.1 \%$

EDTA. Sodium linoleate liposomes were formed through addition of $0.1 \mathrm{M} \mathrm{NaOH}$ to $\mathrm{pH} 10.5$ and the final volume adjusted volumetrically to $50 \mathrm{~mL}$ with borate buffer. The substrate was stored as $5 \mathrm{~mL}$ aliquots under argon at $4{ }^{\circ} \mathrm{C}$ in amber-glass vials until required. (Werber et al., 2011) over a period of 5 hours.

\subsection{Preparation \& Characterisation of the Oxidant}

An aqueous solution $(20 \mathrm{mM})$ of the hydrophilic azo-initiator AAPH was prepared daily in $0.05 \mathrm{M}$ PBS ( $\mathrm{pH} 7.4)$, and stored at $4{ }^{\circ} \mathrm{C}$ until required and on ice during use. The rate constant $\left(k_{1}\right)$ for the unimolecular decomposition of $\mathrm{AAPH}$ was determined at $37^{\circ} \mathrm{C}$ by monitoring the first-order loss of the azo chromophore $\left(\lambda_{\max } 366 \mathrm{~nm} ; \varepsilon_{\max } 22 \mathrm{M}^{-1} \mathrm{~cm}^{-1}\right)$ 


\subsection{Oxidation of Substrate}

The oxidation of linoleate was achieved by addition of $100 \mu \mathrm{L}$ of linoleate substrate to $850 \mu \mathrm{L}$ of PBS $(0.05 \mathrm{M}, \mathrm{pH} 7.4)$ followed by $50 \mu \mathrm{L}$ of $40 \mathrm{mM}$ AAPH in a semi-micro quartz cuvette (final concentrations: linoleate, $1.6 \mathrm{mM} ; \mathrm{AAPH}, 2 \mathrm{mM}$ ). Linoleate was also oxidised in the presence of increasing concentrations of $\operatorname{Trolox}^{1}(2-8 \mu \mathrm{M})$. Since AAPH has a relatively high absorbance under $260 \mathrm{~nm}$, a second cuvette containing only oxidant and buffer was prepared to correct for any absorption change due to the decomposition of the azo compound. The progress of the oxidation was monitored by following the production of conjugated dienes at $234 \mathrm{~nm}$ and $37^{\circ} \mathrm{C}$.

Identical parallel peroxidations were performed in a thermostatically-controlled water bath $\left(37^{\circ} \mathrm{C}\right)$, allowing removal of aliquots of the reaction mixture at various time points. The peroxidation reaction was terminated in these aliquots by the addition of $20 \mu \mathrm{L}$ of BHT ( $5 \mathrm{mM}$ in methanol), and the concentration of the two major linoleate hydroperoxides, E,E-9HPODE and E,E-13-HPODE, determined by reversed-phase HPLC (Perkin-Elmer Series $200 \mathrm{HPLC}$ ) on a C18 column (Phenomenex) using methanol/ammonium acetate $(10 \mathrm{mM}, \mathrm{pH}$ 5) $(95 / 5 \% \mathrm{v} / \mathrm{v})$ as mobile phase. Eluted hydroperoxides were detected by post-column chemiluminescence using a luminol/peroxidase system (Bowry \& Stocker, 1993).

\subsection{Kinetic Analysis of Data}

Raw data from all experiments was exported in ASCII format and parsed using Microsoft Excel $2007^{2}$ for Windows (Microsoft Cooperation, Redmond, WA, USA). Data were initially evaluated to identify $A_{\max }$ and the rate of oxidation was estimated using the method of finite differences (Eqn. A3), which allowed identification of $v_{\max }, t_{\max }$ and $t_{\text {lag }}$ using conventional methods. Following these initial estimates, experimental data were fitted to

\footnotetext{
${ }^{1} 6$-Hydroxy-2,5,7,8-tetramethylchroman-2-carboxylic acid.

${ }^{2}$ Microsoft Excel 2010 for Windows is now available. The curve fitting procedures have been validated for this version of the software.
} 
1 three empirical sigmoid functions (Eqns. 1 - 3) using the Microsoft Excel Solver to evaluate

2 the curve fitting parameters for each (Bourg, 2005). Further details of the curve fitting procedure are available on request from the corresponding author. To determine selected rate constants, a steady state (Rice-Herzfeld) approach was applied (Wright, 2004), according to the scheme depicted in Fig. 2.

\subsection{Statistical Analysis of Data}

Non-parametrically distributed data were assessed by the Mann Whitney $U$ test or by Kendall rank-order correlation using the Statistics Package for Social Sciences (SPSS) for Windows. Results are given as mean \pm standard deviation unless otherwise stated. $P<$ 0.05 was considered as statistically significant.

\section{Results \& discussion}

\subsection{Kinetics of $A A P H$ decomposition}

AAPH was selected as an initiator of peroxidation in order to obtain a constant and well-characterised rate of chain initiation. The rate of alkyl radical production from AAPH $\left(A^{*}\right.$ in Fig. 2) was determined by following the disappearance of the azo chromophore at $\lambda_{\max }$ $366 \mathrm{~nm}$ (Fig. 3) giving $k_{1}=2.07 \pm 0.18 \times 10^{-6} \mathrm{~s}^{-1}\left(\mathrm{t}_{1 / 2}=93 \mathrm{hrs}\right)$ a value in agreement with that previously reported $\left(1.36 \times 10^{-6} \mathrm{~s}^{-1}\right)($ Niki et al., 1990). The rate of alkylperoxyl generation $\left(R_{1}\right)$ determined by this method was calculated as $4.14 \pm 1.1 \times 10^{-8} \mathrm{M} \mathrm{s}^{-1}$ (for $20 \mathrm{mM} \mathrm{AAPH}$ ), which equates to a rate of chain initiation in the aqueous phase $\left(R_{3, \text { aq }}\right)$ of $3.97 \times 10^{-8} \mathrm{M} \mathrm{s}^{-1}$ (taking $R_{3}=2 \mathrm{e} R_{1}$, assuming an efficiency, e, of 0.48; Rackova et al., 2002).

The use of azo-initiators such as AAPH in lipid peroxidation studies is controversial, principally on the grounds of physiological relevance. However, when undertaking kinetic studies of simple two-component systems (substrate and antioxidant), their use greatly 
1 simplifies kinetic analysis, as it permits a steady-state (Rice-Hertzfeld) treatment of the

2 system. Azo compounds readily undergo thermolysis to produce alkyl radicals which can

3 induce free radical or nucleophilic oxidation, the former through combination with molecular oxygen to produce alkylperoxyl radicals. This goes some way to address the question of biological relevance, as peroxyl radicals play a major role in oxidative stress in vivo (Spiteller, 1998). Recently, Werber and co-workers demonstrated that at low $\mathrm{pH}$, thermal decomposition of $\mathrm{AAPH}$ predominates (producing alkyl radicals), but at $\mathrm{pH} \geq 7$, hydrolysis dominates, producing 2,2'-azobis-(2-carbamoylpropane), which does not undergo thermal decomposition to form alkyl radicals (Werber et al., 2011). These findings may go some way to explain the low phase-transfer efficiency reported for AAPH-mediated peroxidations (typically 28 - $55 \%$; Burton \& Ingold, 1981).

\subsection{Kinetics of linoleate peroxidation}

Data for our curve fitting procedure was generated by following the AAPH-mediated peroxidation of aqueous linoleate liposomes ( \pm Trolox) at $\lambda_{\max } 234 \mathrm{~nm}$, correcting for UV absorbance due to azo decomposition products. As expected, plots of absorbance vs. time had an overall sigmoid appearance, with a dose-responsive increase in lag time with increasing concentrations of Trolox (representative data is shown in Fig. 4). Occasionally, at $0 \mu \mathrm{M}$ Trolox, there was an identifiable lag phase, inconsistent with the kinetic profile expected with azo-initiators. This was due to autooxidation in some aliquots of linoleate, subsequently confirmed by strong absorbance at $3400 \mathrm{~cm}^{-1}$ (hydroperoxides) and $1750 \mathrm{~cm}^{-1}$ (carbonyls) on FT-IR ATR spectra (Thermo Nicolet) (Vlachos et al., 2006) and by measurement of the UV absorbance of the liposomes at $234 \mathrm{~nm}$ (data not shown).

The rate of chain initiation in the lipid phase was determined from the gradient of Fig. 5 (i.e. an inhibition method; Niki et al., 1986), which gave $R_{3, \text { lipid }}=9.44 \times 10^{-9} \mathrm{M} \mathrm{s}^{-1}$, a value in 
1 excellent agreement with that reported by Liu (2006) $\left(7.70 \times 10^{-9} \mathrm{M} \mathrm{s}^{-1}\right)$ for a similar

2 peroxidation system (10.9 $\mathrm{mM}$ linoleate, $20 \mathrm{mM} \mathrm{AAPH})$. Thus, for our system, the phase

3 transfer efficiency $(\eta)$ is in the region of $23 \%$, which in light of the findings of Werber et al.

4 (2011) (described in section 3.1) may be ascribable to the slightly basic pH of our oxidation

system. Similar kinetic data were obtained from measurements of E,E-9-HPODE and E,E13-HPODE formation (not shown).

We selected linoleate as a substrate as its peroxidation is well-characterised, and the final concentration of linoleate used $(1.6 \mathrm{mM})$ would appear to be in keeping with that expected in vivo (2 mM) (Glaser et al., 2010), as well as observing the critical micelle concentration of $1 \mathrm{mM}$ (Fygle \& Melo, 1996). Admittedly, following the formation of conjugated dienes at $\lambda_{\max } 234 \mathrm{~nm}$ is limited in terms of discriminating between the products of oxidation. However, the high signal-to-noise ratio and potential for continuous monitoring make this technique enduring. As a companion to this method, we followed the concomitant formation of E,E-9-HPODE and E,E-13-HPODE by HPLC with chemiluminescent detection, observing a close agreement between the two methods $\left(R^{2} \geq 0.91\right)$, as previously highlighted by Bowry \& Stocker (1993). A superior companion to measurement of conjugated dienes at $\lambda_{\max } 234 \mathrm{~nm}$ would be to adopt a mass spectrometry (MS) approach to measure formation of diverse linoleate oxidation products (e.g. epoxy- and oxo-derivatives). A popular and effective method is $\mathrm{Ag}^{+}$-Coordination lonspray (CIS) MS, which utilizes silver ions' ability to coordinate with double bonds, increasing detection accuracy through the characteristic doublet isotopic pattern of $\left[\mathrm{M}+\mathrm{Ag}^{107}\right]^{+}$and $\left[\mathrm{M}+\mathrm{Ag}^{109}\right]^{+}[$Bayer et al., 1999]. A particularly useful CIS-MS target is to follow the fragmentation of hydroperoxides through Hock cleavage, which can be used to determine the involvement of specific intermediates in lipid oxidation (e.g. bicyclic endoperoxides) [Yin et al., 2005]. 


\subsection{Sigmoid models of linoleate peroxidation}

We sought to determine whether a classic sigmoid function could adequately model linoleate peroxidation, and whether such modelling could be determined without the aid of dedicated curve-fitting software. To achieve this, kinetic data were exported to Microsoft Excel and fitted to each of the three sigmoid functions described in section 1 (Eqns. 1 - 3) using the Solver add-in. Visual inspection of Fig. 6 shows that the curve predicted by the Richards' function intersected each data point of the observed data, and easily accommodated the asymmetry associated with lipid peroxidation curves. This was supported by correlation analysis (observed vs. calculated) in which the Richards' equation had a much stronger Kendall rank-order correlation coefficient $\left(R^{2} \geq 0.998 ; P<0.005\right)$ than other models (Table 1).

The Microsoft Excel Solver evaluates curve fitting parameters using a GRG2 algorithm, utilizing Newton-Raphson iterations to determine the root of the gradient of the function (Flystra et al., 1998). A drawback to this procedure is that initial guesses for each parameter must be provided. For the Prout-Tompkins and Gompertz functions, these parameters were easily approximated from the respective equations (Eqns. A5 - A6). For the Richards' function, the curve fitting parameters have no physicochemical meaning (other than $a$, the maximum absorbance) and supplying initial guesses for these parameters was by trial and error. Exemplar curve fitting parameters and curvilinear equations are given in Table 2.

.

On the basis of these findings, the Richards' function (Eqn. 2) was partially reparameterised (Eqn. 4) and differentiated with respect to time to provide an expression for the rate of oxidation (Eqn. 5).

$$
A=a[1+b \exp (-c t)]^{1 /(1-d)}
$$




$$
\frac{d A}{d t}=\frac{c A}{(d-1)\left[1-(A / a)^{d-1}\right]}
$$

1

2 To obtain the point of inflection, the second derivative was obtained (Eqn. A7), which equals

$$
t_{\max }=\left(\frac{1}{b}\right)\left[\ln \frac{b}{(c-1)}\right]
$$

$$
\tau=\frac{A_{t \max }-\left(v_{\max } \cdot t_{\max }\right)}{v_{\max }}
$$

We propose that the solution to Eqn. 8, the time $(\tau)$ corresponding to the maximum of the second derivative, is a suitable alternative definition of lag time (the $x$-intercept of a tangent to $\left.v_{\max }\right)$. The two parameters have common mathematical origins: lag time can be considered to correspond to the rapid onset of peroxidation, which on a plot of $d^{2} A / d t^{2} v s$. $t$, will have a maximum corresponding to lag time, and therefore $\tau$. The relationship between these two parameters is sufficiently strong $\left(R^{2}=0.999\right)$ to make such a redefinition compelling. Evaluating lag time in this manner removes subjective errors introduced by graphical determination, and can be extended to include inhibition time ( $\left.t_{\text {inhb }}\right)$, a common kinetic measure in antioxidant studies. 
To validate our approach against existing methods, we evaluated a number of kinetic

parameters using established methods (finite differences, graphical determination of lag time and classical steady-state equations ${ }^{3}$ ) and Eqns. 4 - 8. Results (Table 3) demonstrate that, on average, kinetic parameters obtained from the Richards' function are in good agreement with those obtained from the established methods. The trend in the value of the kinetic constants with increasing concentrations of Trolox is in keeping with that reported by Niki et al. (1986), specifically a decrease in the rate of propagation and kinetic chain length with increasing concentrations of Trolox. More importantly, however, is the fact that the kinetic data calculated from the Richards' function is in excellent agreement with that obtained from established steady state equations.

The Richards' function has been criticised for a lack of physicochemical or biological meaning to the $d$-parameter (Tjørve \& Tjørve, 2010), although the presence of four independent coefficients leads to considerable flexibility, enabling modelling of complex processes. In this respect, it is similar to the Boltzmann equation (vide infra) which has been used to model haemolysis of red blood cells by AAPH (Tang \& Liu, 2007). In this current investigation, the $d$-parameter appears to be critical, as it predicts the early exponential slope marking progression from the initiation phase to the propagation phase. Given that our proposed definition of lag time corresponds to the transition between these two phases, it is crucial that this region of the peroxidation curve is modelled accurately. More generally, the ability of the Richards' function to accommodate the asymmetry associated with kinetic profiles of lipid peroxidation sets it apart from the other models investigated. That in mind, it would be interesting to examine the association between the Richards' parameters and the concentration of oxidant, as this latter factor can substantially alter the kinetic profile of lipid peroxidation; e.g. the biphasic profiles obtained at low concentrations of oxidant (Ziouzenkova et al., 1998).

\footnotetext{
${ }^{3}$ These calculations were performed using MATLAB (MathWorks, Natick, MA, USA).
} 


$$
y=\frac{\Delta y}{\left\{[1+\exp (x-\tau) / d x]+y_{\text {final }}\right\}}
$$

1

\subsection{Further Evaluation of Equations 4-8}

To explore the wider applicability of Eqns. 4-8, we applied the curve fitting process to previously collected data for the oxidation of VLDL (McEneny et al., 1997), LDL (McDowell et al., 1995) and HDL (McPherson et al., 2007) by aqueous copper(II) ions. The kinetic measures of lipid peroxidation were evaluated using commercially available software (SoftMax Pro, Molecular Devices Crop.), which uses finite differences, and by Eqns. 4 - 8.

The kinetic plot for VLDL oxidation (Figure 7A) was characterised by an initial dip in UV absorbance, due to aggregation of the lipoproteins and subsequent Rayleigh scattering, which the Richards' function could not model; however, this did not affect the determination of the kinetic constants $\left(v_{\max }=1.36 \mathrm{nM} \mathrm{s}^{-1} ; t_{\max }=182 \mathrm{~min} ; \tau=137 \mathrm{~min}\right)$. Similarly, the decomposition phase for LDL (Figure 7B) was not modelled by Richards' function ( $v_{\max }=$ $\left.4.38 \mathrm{nM} \mathrm{s}^{-1} ; t_{\max }=112 \mathrm{~min} ; \tau=79 \mathrm{~min}\right)$. The kinetic plot for HDL was more hyperbolic in its overall form, which resulted in a poorer fit at the start and finish of the propagation phase (Figure 7C); this did not affect determination of kinetic constants $\left(v_{\max }=1.90 \times \mathrm{nM} \mathrm{s}^{-1} ; t_{\max }=\right.$ $56 \min ; \tau=31 \mathrm{~min})$.

The peroxidation of lipoproteins is mechanistically more complex than that of simple liposomes, largely because of the wide variety of antioxidant species present, but also due to the varying lipid content. The nature of the oxidant is also crucial, as widely different kinetic profiles are obtained when copper(II), AAPH, haemin or myleoperoxidase are used to initiate oxidation (McPherson et al., unpublished). Despite this, the Richards' function adequately modelled copper(II)-mediated lipoprotein peroxidation, and produced estimates of $v_{\max }, t_{\max }$ and lag time in agreement with those established in the literature (Schnitzer et al., 1995). 
1 Thus, the curve fitting procedure we propose would seem to have a wide applicability to a

2 variety of physicochemical studies.

3

4 4. Conclusion

We propose a model of lipid peroxidation based on a partial reparameterization of the

6 Richards five-parameter logistic function, which does not rely on numerical methods of

7 analysis, and can easily be performed using ubiquitous software such as Microsoft Excel.

8 This approach enables evaluation of $v_{\max }, t_{\max }$ and lag time using only the four curve fitting

9 parameters $a, b, c$ and $d$, and led to a redefinition of the commonly employed kinetic

10 measure lag time, generally regarded as the $x$-intercept of a tangent to $v_{\max }$, but what we

11 define as the time at which the second derivative is maximal. Overall, our approach is

12 simple and accurate, and is widely applicable not only to investigations of lipid/lipoprotein

13 oxidation, but more widely to any kinetic system exhibiting sigmoid reaction curves.

\section{Acknowledgements}

The authors would like to thank Clarke Stevenson and Mervyn Cairnduff for excellent

17 technical support. $\mathrm{AB}$ and $\mathrm{KAC}$ received support from the Department of Employment \&

Learning for Northern Ireland during the completion of this work. 


\section{References}

Antolovich, M., Prenzler, P.D., Patsalides, E., McDonald, S., Robards, K., 2002. Methods for testing antioxidant activity. Analyst 127, 183-198.

Antunes, F., Salvador, A., Marinho, H.S., Alves, R., Pinto, R.E., 1996. Lipid peroxidation in mitochondrial inner membranes. I. An integrative kinetic model. Free Radic. Biol. Med. 21, 917-943.

Bayer, E., Gfrorer, P., Rentel, C, 1999. Coordination-ionspray-MS (CIS-MS), a universal detection and characterization method for direct coupling with separation techniques. Angew. Chem. Int. Ed., 38, 992-995.

Bourg, D.M., 2005. Excel scientific and engineering cookbook. O'Reilly, California.

Bowry, V.W., Stocker, R. 1993. Tocopherol-mediated peroxidation. The prooxidant effect of vitamin $\mathrm{E}$ on the radical-initiated oxidation of human low-density lipoprotein. J. Am. Chem. Soc. 115, 6029-6044.

Brown, M.E., Glass, B.D., 1999. Pharmaceutical applications of the Prout-Tompkins rate equation. Int. J. Pharm. 190, 129-137.

Burton, G.W., Ingold, K.U., 1981. Autoxidation of biological molecules. 1. Antioxidant activity of vitamin $E$ and related chain-breaking phenolic antioxidants in vitro. J. Am. Chem. Soc. $103,6472-6477$.

Cadenas, E., Sies, H., 1998. The lag phase. Free Radic. Res. 28, 601-609.

Dalgaard, P., Koutsoumanis, K., 2001. Comparison of maximum specific growth rates and lag times estimated from absorbance and viable count data by different mathematical models. J. Microbiol. Methods 43, 183-196.

Esterbauer, H., Striegl, G., Puhl, H., Rotheneder, M., 1989. Continuous monitoring of in vitro oxidation of human low density lipoprotein. Free Radic. Res. Commun. 6, 67-75.

Foubert, I., Dewettinck, K., Vanrolleghem, P.A., 2003. Modelling of the crystallization kinetics of fats. Trends Food Sci. Technol. 14, 79-92.

Fygle, K.E., Melo, T.B., 1996. Optical absorption studies of the kinetics of UV- and selfinitiated autoxidation of linoleate micelles. Chem. Phys. Lipids 79, 39-46.

Fylstra, D., Leon Lasdon, Watson, J., Waren, A., 1998. Design and Use of the Microsoft Excel Solver. Interfaces 28, 29-55.

Gieseg, S.P., Esterbauer, H., 1994. Low density lipoprotein is saturable by pro-oxidant copper. FEBS Lett. 343, 188-194.

Giraldo, J., Vivas, N.M., Vila, E., Badia, A., 2002. Assessing the (a)symmetry of concentration-effect curves: empirical versus mechanistic models. Pharmacol. Ther. 95, 2145.

Glaser, C., Demmelmair, H., Koletzko, B., 2010. High-throughput analysis of total plasma fatty acid composition with direct in situ transesterification. PLoS One 5, 1-6. 
Gompertz, B., 1825. On the Nature of the Function Expressive of the Law of Human Mortality, and on a New Mode of Determining the Value of Life Contingencies. Philosophical Transactions of the Royal Society of London 115, 513-583.

Herney-Ramirez, J., Silva, A.M.T., Vicente, M.A., Costa, C.A., Madeira, L.M., 2011. Degradation of Acid Orange 7 using a saponite-based catalyst in wet hydrogen peroxide oxidation: Kinetic study with the Fermi's equation. Applied Catalysis B: Environmental 101, 197-205.

Leith, J.H., Fischer, P.R., Heins, R.D., 1996. A phasic model for the analysis of sigmoid patterns of growth. Acta Hortic 417, 113-118.

Liu, Z.Q., 2006. Icariin: a special antioxidant to protect linoleic acid against free-radicalinduced peroxidation in micelles. J Phys Chem A 110, 6372-6378.

McDowall, L.M., Dampney, R.A.L., 2006. Calculation of threshold and saturation points of sigmoidal baroreflex function curves. Am. J. Physiol. Heart Circ. Physiol. 291, H2003H2007.

McDowell, I.F., McEneny, J., Trimble, E.R., 1995. A rapid method for measurement of the susceptibility to oxidation of low-density lipoprotein. Ann. Clin. Biochem. 32, 167-174.

McEneny, J., Loughrey, C.M., McNamee, P.T., Trimble, E.R., Young, I.S., 1997.

Susceptibility of VLDL to oxidation in patients on regular haemodialysis. Atherosclerosis 129 , 215-220.

McPherson, P.A., Young, I.S., McEneny, J., 2007. A dual role for lecithin:cholesterol acyltransferase (EC 2.3.1.43) in lipoprotein oxidation. Free Radic. Biol. Med. 43, 1484-1493.

Molinari, N., Morena, M., Cristol, J.P., Daure, J.P., 2002. Free knot splines for biochemical data. Comput. Methods Programs Biomed. 67, 163-167.

Morton, K.W., Mayer, D.F., 2005. Numerical solutions of partial differential equations: An introduction. Cambridge University Press, England.

Niki, E. 1990. Free radical initiators as source of water- or lipid-soluble peroxyl radical. Methods Enzymol. 186: 100-108.

Niki, E., Saito, M., Yoshikawa, Y., Yamamoto, Y., Kamiya, Y., 1986. Oxidation of lipids. XII. Inhibition of oxidation of soybean phosphatidylcholine and methyl linoleate in aqueous dispersions by uric acid. Bull. Chem. Soc. Jpn. 59, 471-477.

Pinchuk, I., Lichtenberg, D., 2002. The mechanism of action of antioxidants against lipoprotein peroxidation, evaluation based on kinetic experiments. Prog. Lipid Res. 41, 279314.

Porter, N.A., Lehman, L.S., Weber, B.A., Smith, K.J., 1981. Unified mechanism for polyunsaturated fatty acid autoxidation. Competition of peroxy radical hydrogen atom abstraction, .beta.-scission, and cyclization. J. Am. Chem. Soc. 103, 6447-6455.

Porter, N.A., Mills, K.A., Carter, R.L., 1994. A Mechanistic Study of Oleate Autoxidation: Competing Peroxyl H-Atom Abstraction and Rearrangement. J. Am. Chem. Soc. 116, 66906696. 
Rackova, L., Stefek, M., Majekova, M., 2002. Structural aspects of antioxidant activity of substituted pyridoindoles. Redox Rep. 7, 207-214.

Raveh, O., Pinchuk, I., Schnitzer, E., Fainaru, M., Schaffer, Z., Lichtenberg, D., 2000. Kinetic analysis of copper-induced peroxidation of HDL, autoaccelerated and tocopherol-mediated peroxidation. Free Radic. Biol. Med. 29, 131-146.

Richards, F.J., 1959. A flexible growth function for empirical use. J. Exp. Bot. 10, 290-300.

Schneider, M., Jentzsch, A.M., Trommer, W.E., Biesalski, H.K., 1998. EPR kinetic studies of the LDL oxidation process driven by free radicals. Free Radic. Res. 28, 451-458.

Schnitzer, E., Pinchuk, I., Fainaru, M., Schafer, Z., Lichtenberg, D. 1995. Copper-induced lipid oxidation in unfractionated plasma: the lag preceding oxidation as a measure of oxidation-resistance. Biochem. Biophys. Res. Commun. 216, 854-861.

Sevanian, A., Hochstein, P., 1985. Mechanisms and consequences of lipid peroxidation in biological systems. Annu. Rev. Nutr. 5, 365-390.

Simon, L., Nazmul Karim, M., 2001. Probabilistic neural networks using Bayesian decision strategies and a modified Gompertz model for growth phase classification in the batch culture of Bacillus subtilis. Biochem. Eng. J. 7, 41-48.

Spiteller, G., 1998. Linoleic acid peroxidation--the dominant lipid peroxidation process in low density lipoprotein--and its relationship to chronic diseases. Chem. Phys. Lipids 95, 105-162.

Surrey, K., 1964. Spectrophotometric Method for Determination of Lipoxidase Activity. Plant Physiol. 39, 65-70.

Tang, Y.Z., Liu, Z.Q., 2007. Free-radical-scavenging effect of carbazole derivatives on AAPH-induced hemolysis of human erythrocytes. Bioorg. Med. Chem. 15, 1903-1913.

Tjørve, E., Tjørve, K.M.C., 2010. A unified approach to the Richards-model family for use in growth analyses: Why we need only two model forms. J. Theor. Biol. 267, 417-425.

Verhulst, P.F., 1838. A note on population growth. Correspondence Mathematiques et Physiques 10, 113-121.

Vlachos, N., Skopelitis, Y., Psaroudaki, M., Konstantinidou, V., Chatzilazarou, A., Tegou, E., 2006. Applications of Fourier transform-infrared spectroscopy to edible oils. Anal. Chim. Acta 573-574, 459-465.

Werber, J., Wang, Y.J., Milligan, M., Li, X., Ji, J.A., 2011. Analysis of 2,2'-azobis (2amidinopropane) dihydrochloride degradation and hydrolysis in aqueous solutions. J. Pharm. Sci. 100, 3307-3315.

Wright, M.R. 2004. An introduction to chemical kinetics. England, Wiley.

Yin, H., Musiek, E., Gao, L., Porter, N. A., Morrow, J. D. 2005. Regiochemistry of neuroprostanes generated from the peroxidation of docosahexaenoic acid in vitro and in vivo. J. Biol. Chem., 280, 26600-26611. promote oxidation of LDL by markedly different mechanisms. Free Radic. Biol. Med. 24, 607623. 
2 Curve fitting statistics for sigmoid models of linoleate peroxidation at different 3 concentrations of Trolox ${ }^{a}$.

4

\begin{tabular}{ccccccc}
\hline & \multicolumn{2}{c}{ Prout-Tompkins } & \multicolumn{2}{c}{ Richards } & \multicolumn{2}{c}{ Gompertz } \\
[Trolox] $/ \mu \mathrm{M}$ & $\boldsymbol{R}^{2}$ & $\boldsymbol{R S S}$ & $\boldsymbol{R}^{2}$ & $\boldsymbol{R S S}$ & $\boldsymbol{R}^{2}$ & $\boldsymbol{R S S}$ \\
\hline 0.0 & 0.9940 & 0.2678 & 0.995 & 0.082 & 0.9998 & 0.0048 \\
2.0 & 0.9729 & 1.1204 & 1.000 & 0.000 & 0.9847 & 0.2985 \\
4.0 & 0.9560 & 2.2296 & 1.000 & 0.000 & 0.9641 & 0.8892 \\
6.0 & 0.9278 & 3.7978 & 1.000 & 0.000 & 0.9296 & 1.9414 \\
8.0 & 0.9068 & 4.9625 & 1.000 & 0.000 & 0.9020 & 2.7575
\end{tabular}

abserved data vs. modelled data; $P<0.001, n=6$ in all such cases; $R^{2}$, Kendall rank-order correlation coefficient; $R S S$, relative sum of squares. 


\section{TABLE 2}

Curve fitting parameters for Richards' function when applied to linoleate peroxidation.

\section{Curve fitting parameters}

\begin{tabular}{cccccc} 
[Trolox] $/ \mu \mathrm{M}$ & $\boldsymbol{a}$ & $\boldsymbol{b}$ & $\boldsymbol{c}$ & $\boldsymbol{d}$ & \\
\hline 0.0 & 0.9751 & 1410870 & 0.2252 & 6.365 & $A=0.9751\left(1410871 \exp ^{-0.2252 t}\right)^{-0.1864}$ \\
2.0 & 0.9681 & 1410870 & 0.1865 & 5.662 & $A=0.9681\left(1410871 \exp ^{-0.1865 t}\right)^{-0.2145}$ \\
4.0 & 0.9617 & 1410870 & 0.1653 & 4.992 & $A=0.9617\left(1410871 \exp ^{-0.1653 t}\right)^{-0.2505}$ \\
6.0 & 0.9680 & 1410870 & 0.1540 & 3.907 & $A=0.9680\left(1410871 \exp ^{-0.1540 t}\right)^{-0.3441}$ \\
8.0 & 0.9618 & 1410870 & 0.1399 & 3.496 & $A=0.9618\left(1410871 \exp ^{-0.1399 t}\right)^{-0.4006}$ \\
\hline
\end{tabular}




\section{TABLE 3}

Comparison of kinetic measures of linoleate peroxidation ${ }^{a}$.

\begin{tabular}{|c|c|c|c|c|c|c|c|c|c|c|}
\hline \multirow[b]{2}{*}{ [Trolox] $/ \mu \mathrm{M}$} & \multicolumn{2}{|c|}{$t_{\max } / \min$} & \multicolumn{2}{|c|}{ Lag time /min } & \multicolumn{2}{|c|}{$v_{\max } \times 10^{-8} / \mathrm{M} \mathrm{s}^{-1}$} & \multicolumn{2}{|c|}{$R_{5} \times 10^{-8} / \mathrm{M} \mathrm{s}^{-1}$} & \multicolumn{2}{|c|}{$k c l$} \\
\hline & $(i)^{b}$ & $(\mathrm{ii})^{c}$ & (i) & (ii) & (i) & (ii) & (i) & (ii) & (i) & (ii) \\
\hline 0.0 & 40 & 55 & 24 & 27 & 6.90 & 8.28 & 6.29 & 7.04 & 9.8 & 10.9 \\
\hline 2.0 & 60 & 68 & 32 & 37 & 6.89 & 8.10 & 5.20 & 4.03 & 8.1 & 8.7 \\
\hline 4.0 & 68 & 77 & 44 & 47 & 6.14 & 7.90 & 4.15 & 3.48 & 6.5 & 7.3 \\
\hline 6.0 & 80 & 85 & 52 & 60 & 5.91 & 7.45 & 3.68 & 5.57 & 5.7 & 6.3 \\
\hline 8.0 & 92 & 95 & 64 & 70 & 5.08 & 7.22 & 3.32 & 4.70 & 5.2 & 5.4 \\
\hline
\end{tabular}

${ }^{a}[$ Linoleate $]=1.16 \mathrm{mM} ;[\mathrm{AAPH}]=2.0 \mathrm{mM} ; R_{1}=4.9 \times 10^{-9} \mathrm{M} \mathrm{s}^{-1} ; R_{3, \text { lipid }}=6.44 \times 10^{-9} \mathrm{M} \mathrm{s}^{-1} ; R_{5}=$ rate of propagation; $\mathrm{kcl}=$ kinetic chain length $=R_{5} / R_{3}$.

${ }^{b}$ (i) finite differences/graphical method.

'(ii) Richards' function. 


\section{FIGURE LEGENDS}

Fig. 1 Kinetic profiles of lipid peroxidation. A. Following conjugated diene formation at $\lambda_{\max } 234 \mathrm{~nm}$ produces a plot in which maximum absorbance is achieved at $A_{\max }$. Approximately halfway along the propagation slope, $v_{\max }$ is attained, which has a corresponding value on the abscissa, $t_{\max } ; t_{\mathrm{lag}}$ is evaluated as the $x$-intercept of a tangent to $v_{\max }$. B. A plot of the first derivative $(\mathrm{dA} / \mathrm{dt} \longrightarrow)$ shows the rate of peroxidation as a function of time and has a maxima at $v_{\max }$. A plot of the second derivative $\left(d^{2} A / d t^{2} \cdots \cdots \cdot\right)$ shows the time dependency of the rate of peroxidation and has a maxima at $\tau$ and a first root equal to $t_{\max }$.

Fig. 2 Steady state (Rice-Hertzfeld) treatment of lipid peroxidation. Thermolysis of AAPH produces alkyl radicals $\left(\mathrm{A}^{\bullet} ; k_{1}=1.36 \times 10^{-6} \mathrm{M}^{-1} \mathrm{~s}^{-1}\right)$, which rapidly combine with $\mathrm{O}_{2}$ to form alkylperoxyl radicals $\left(\mathrm{AOO} * k_{2}=1 \times 10^{9} \mathrm{M}^{-1} \mathrm{~s}^{-1}\right)$, subsequently combining with linoleate in the initiation phase of peroxidation, forming linoleate radicals $\left(k_{3}=6 \times\right.$ $\left.10^{1} \mathrm{M}^{-1} \mathrm{~s}^{-1}\right)$. Linoleate radicals react with $\mathrm{O}_{2}$, forming linoleate peroxyl radicals $\left(k_{4}=1\right.$ $\left.\times 10^{9} \mathrm{M}^{-1} \mathrm{~s}^{-1}\right)$, which can react with further linoleate molecules to form additional linoleate radicals $\left(k_{5}=6 \times 10^{1} \mathrm{M}^{-1} \mathrm{~s}^{-1}\right)$. Alternatively, a termination reaction can occur through biradical quenching or through the action of a chain-breaking antioxidant, e.g. Trolox $\left(k_{7}=2 \times 10^{7} \mathrm{M}^{-1} \mathrm{~s}^{-1}\right)$.

Fig. 3 Decomposition of AAPH at $37^{\circ} \mathbf{C}$. The first order loss of the azo chromophore was followed at $\lambda_{\max } 366 \mathrm{~nm}$. The gradient of the graph $=k_{1}=2.07 \times 10^{-6} \mathrm{~s}^{-1}$.

Fig. 4 Typical kinetic profiles of linoleate peroxidation. Peroxidation of linoleate (1.6 $\mathrm{mM})$ by $\mathrm{AAPH}(2 \mathrm{mM})$ in the absence and presence of increasing concentrations of Trolox $(0-8 \mu \mathrm{M})$ was monitored at $\lambda=234 \mathrm{~nm}$ for 2.5 hours. An obvious doseresponse effect was observed with increasing concentrations of Trolox. Results shown are those typical of such an experiment. Figure legend: $0 \mu \mathrm{M}$ Trolox $\boldsymbol{\bullet} ; 2 \mu \mathrm{M}$ Trolox $\bullet ; 4 \mu \mathrm{M}$ Trolox $\Delta ; 6 \mu \mathrm{M}$ Trolox $\bullet$; and $8 \mu \mathrm{M}$ Trolox $*$.

Fig. 5 Plot of lag time as a function of [Trolox]/[AAPH]. The gradient of the graph = $R_{3, \text { lipid }}=9.44 \times 10^{-9} \mathrm{M} \mathrm{s}^{-1}$.

Fig. 6 Comparison of sigmoid models of linoleate peroxidation. Observed data (open circles, $\bigcirc)$ were co-plotted with data calculated from the Prout-Tompkins equation (dashed line ------), the Richards equation (solid line _-) and the Gomperz equation (dotted line …....). In all instances, the Richards' equation provided the best fit for the observed data. Data shown are for the peroxidation of linoleate (1.6 mM) by AAPH (2 $\mathrm{mM})$ in the presence of $6 \mu \mathrm{M}$ Trolox and are typical of those obtained.

Fig. 7 Curve fitting of the Richards' function for peroxidation of VLDL, LDL and HDL. Data for peroxidation of VLDL (A), LDL (B) and HDL (C) was achieved as described and the observed data (open circles, $\bigcirc)$ co-plotted with data calculated by applying the Richards' equation (solid line - - ). 


\section{APPENDIX}

\section{Approximating Derivatives using Finite Differences}

By definition, the derivative of a function is given by:

$$
f^{\prime}(x)=\lim _{h \rightarrow 0} \frac{f(x+h)-f(x)}{h}
$$

The first derivative can be approximated using so-called finite differences to determine the gradient of an adjacent secant line:

$$
f^{\prime}(x) \approx \frac{f(x+\delta x)-f(x)}{\delta x}
$$

Equation (A2) is better approximated using the central difference approach, which smoothes the derivative at a particular point by taking into account the value on either side of the function:

$$
f^{\prime}(x)=\frac{f\left(x_{i+1}\right)-f\left(x_{i-1}\right)}{2 \delta x}
$$

An extension of this approach is to use the five-point formula, which smoothes the derivative by taking into account a greater number of points on either side of the point under consideration:

$$
f^{\prime}(x)=\frac{1}{12 h}\left[f\left(x_{i-2}\right)-8 f\left(x_{i-1}\right)+8 f\left(x_{i+1}\right)-f\left(x_{i+2}\right)\right.
$$

\section{Determining Initial Guesses for the Curve Fitting Parameters for Equations 1 and 2}

Equations 1 and 2 can be rearranged (Eqn. A5 and A6) to enable evaluation of the curvature constant for each value of $A$ and $t$; the average $b$ is then used as an initial guess for the Solver programme.

$$
\begin{gathered}
b=\frac{\ln \left(A / A_{\max }\right)}{t-t_{\text {max }}} \\
b=\frac{\ln \left[\ln \left(A_{\max } / A\right)\right]}{t-t_{\max }}
\end{gathered}
$$

\section{Second Derivative of Equation 5}

$$
\frac{d^{2} A}{d x^{2}}=\frac{c f^{\prime}}{(d-1)\left[1-d\left(A / A_{\max }\right)^{d-1}\right]}
$$


A

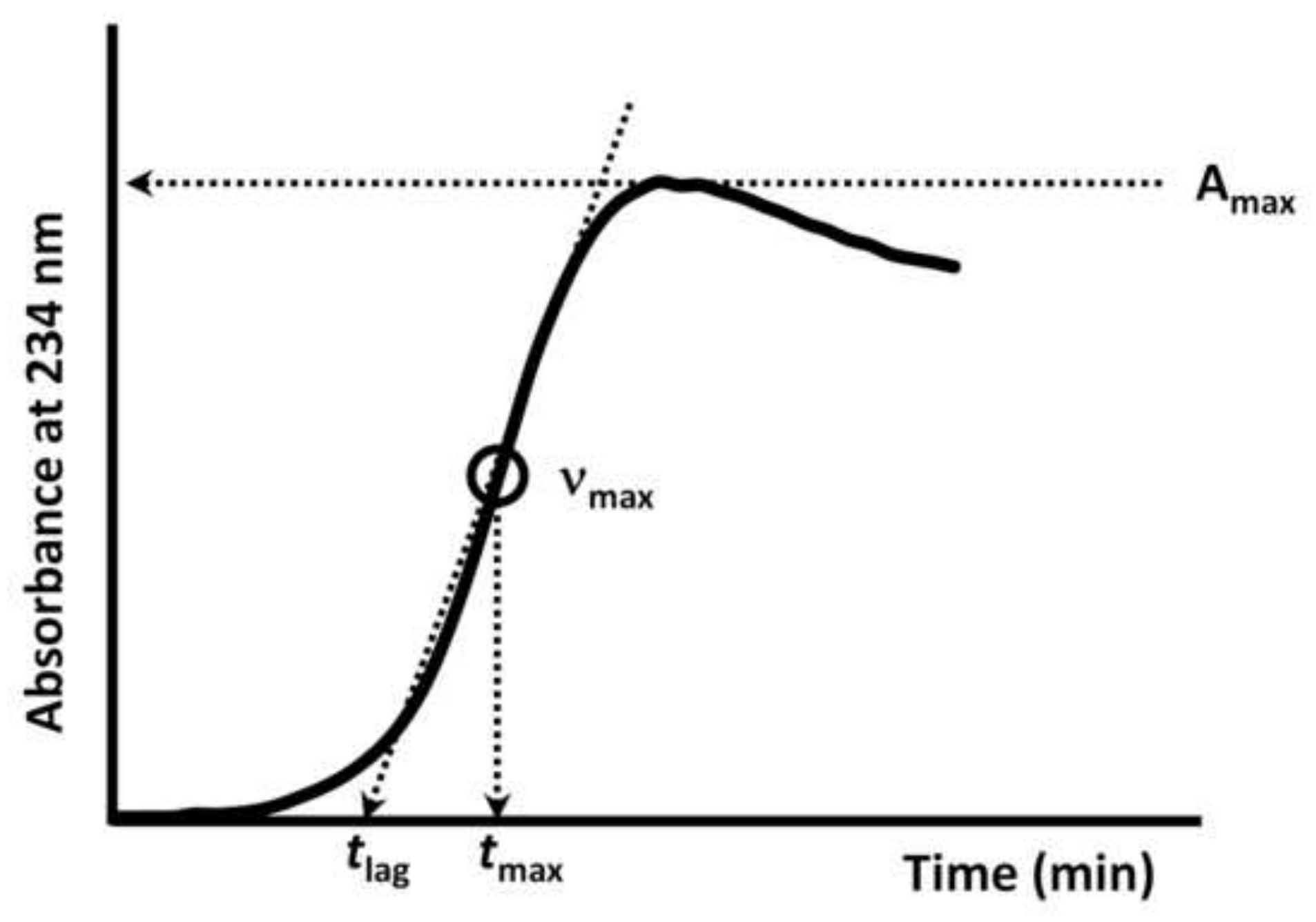


B

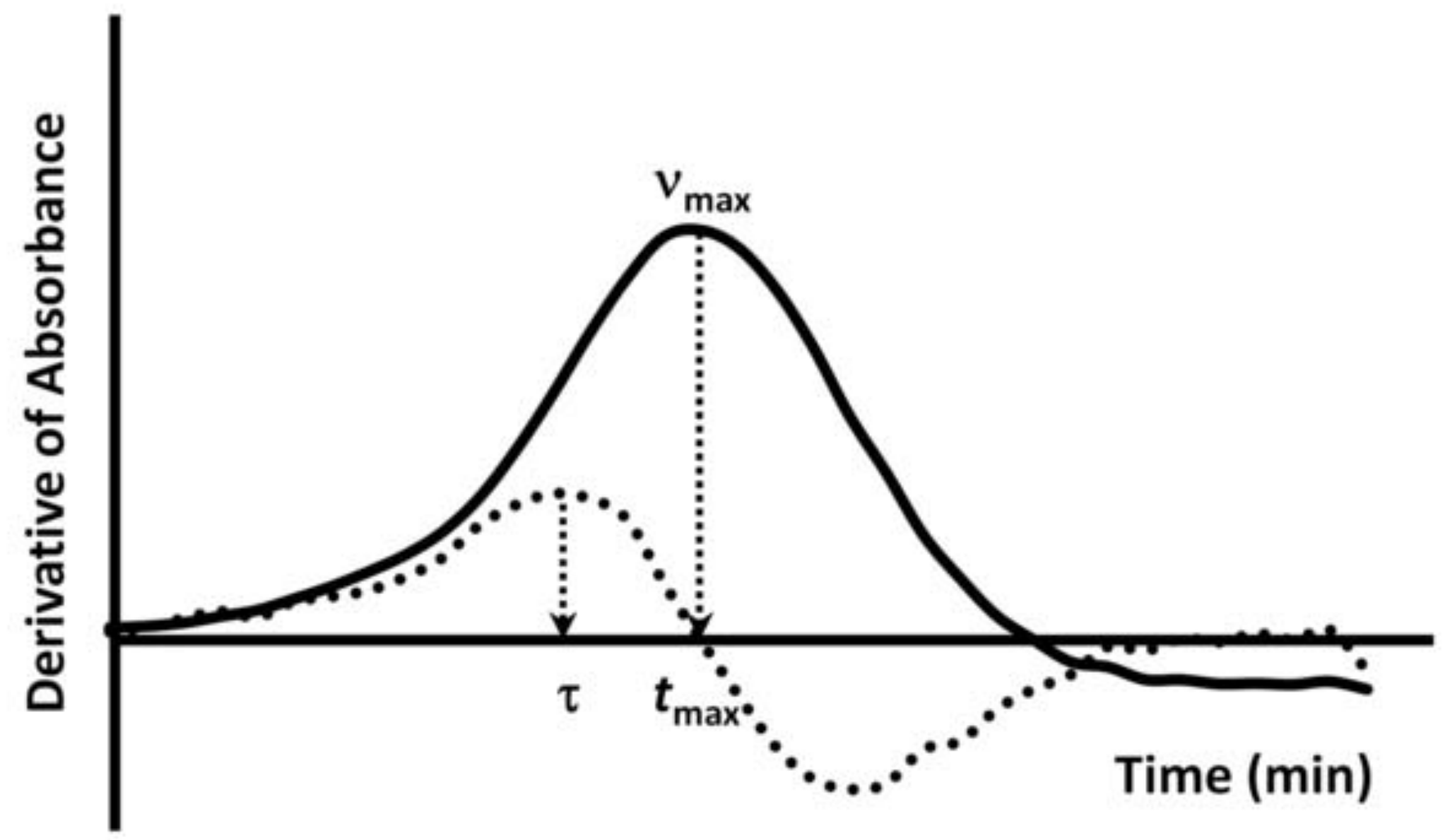




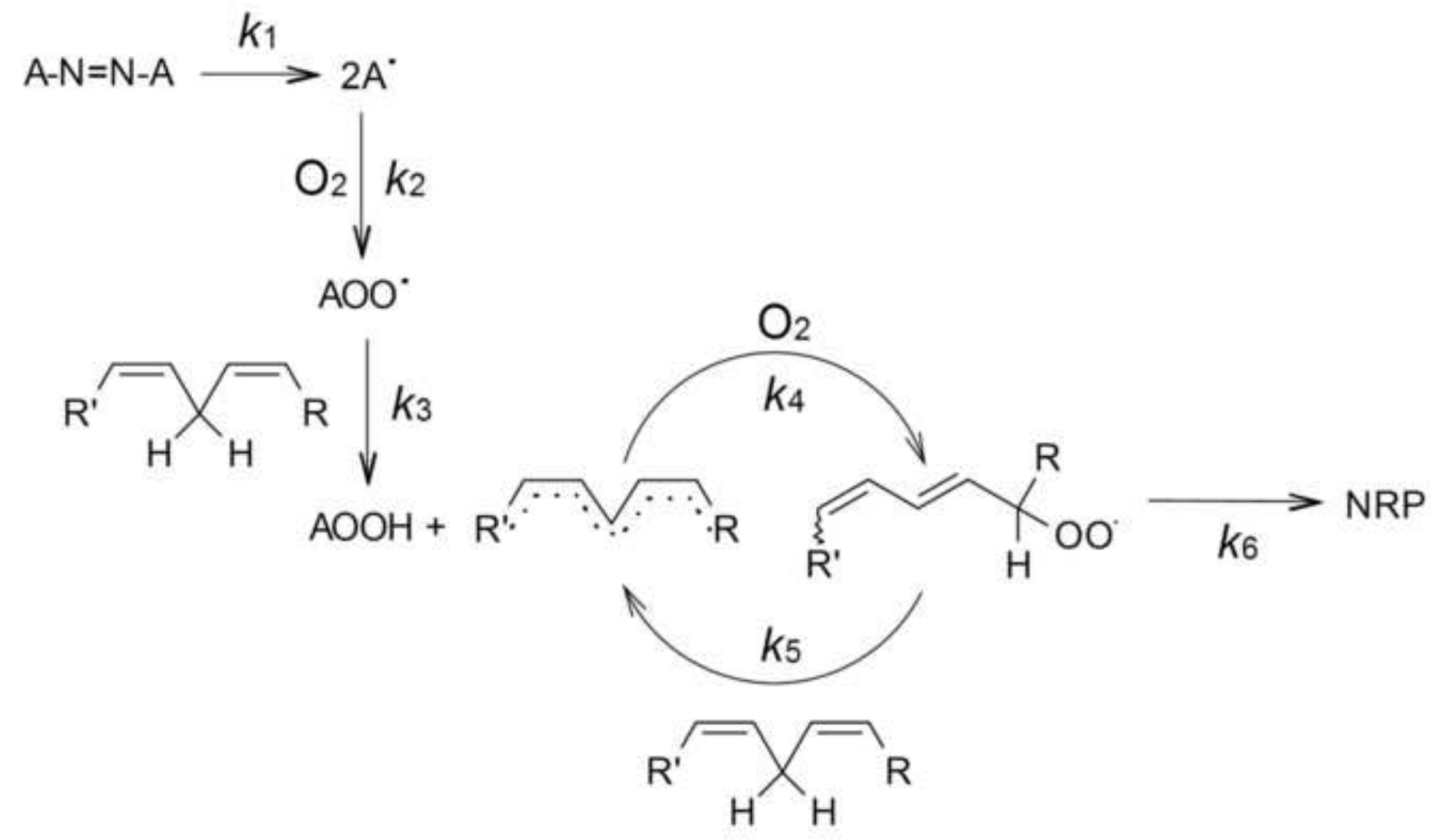




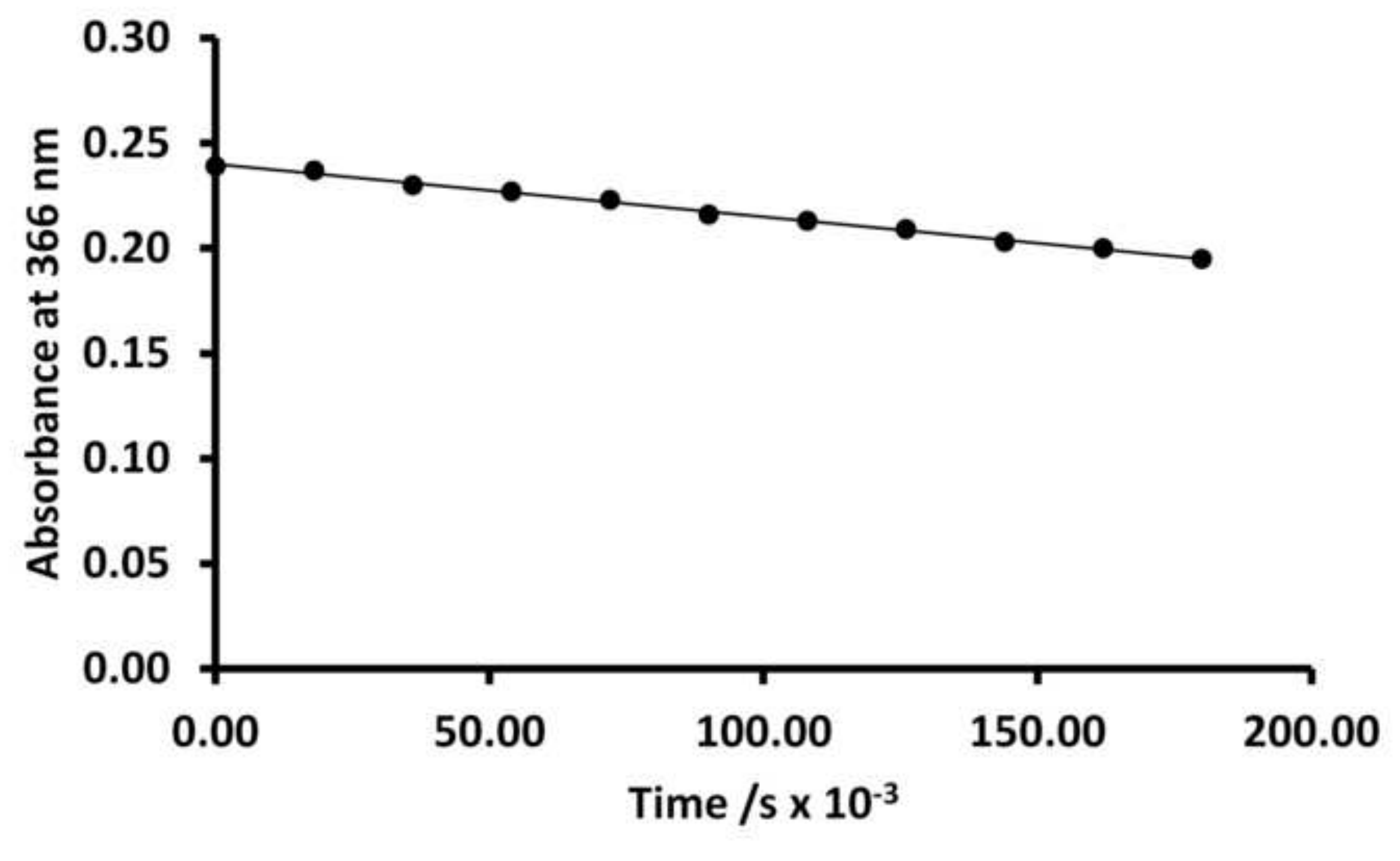

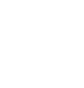

Time $/ \mathrm{s} \times 10^{-3}$

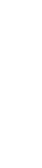

(




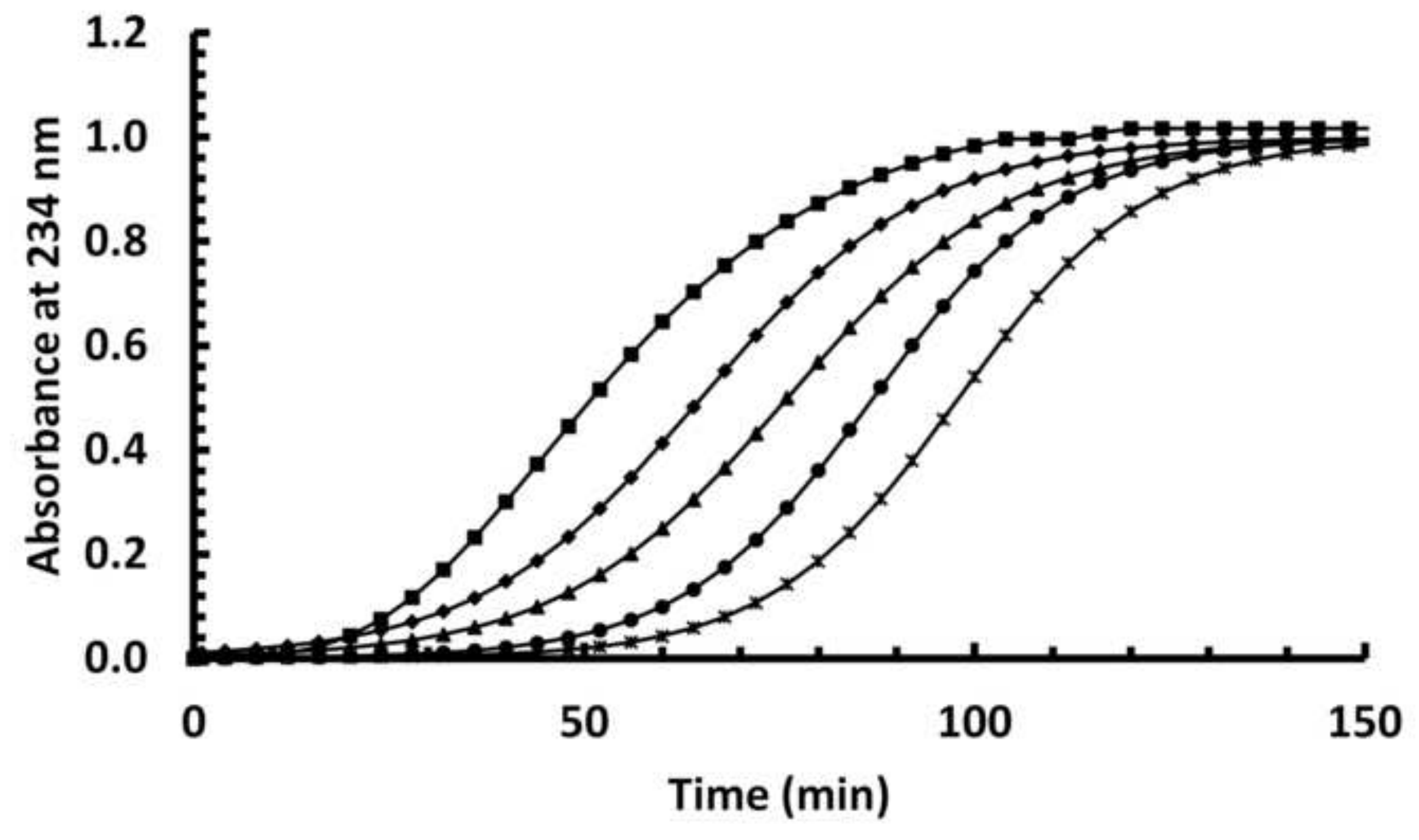




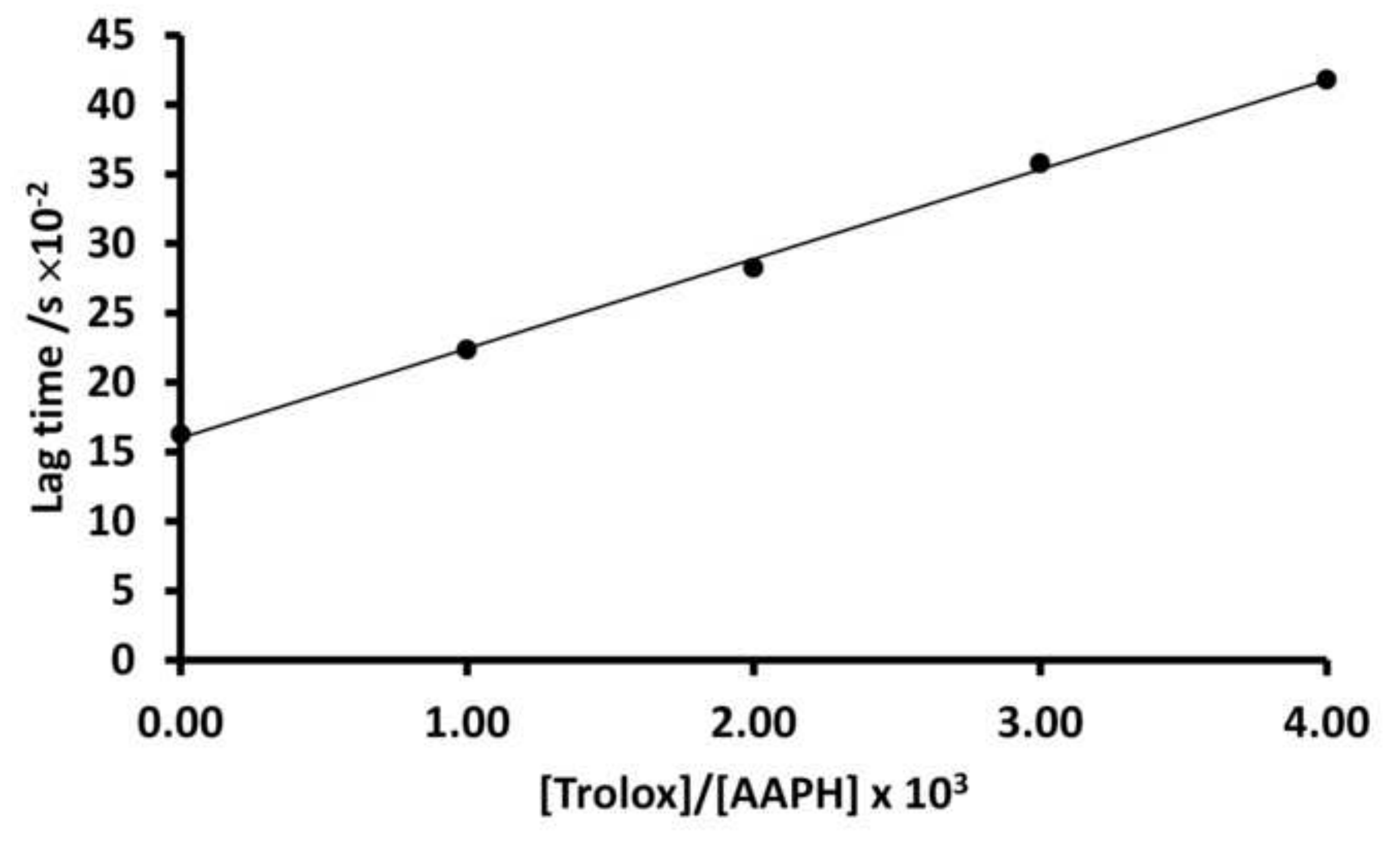

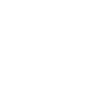




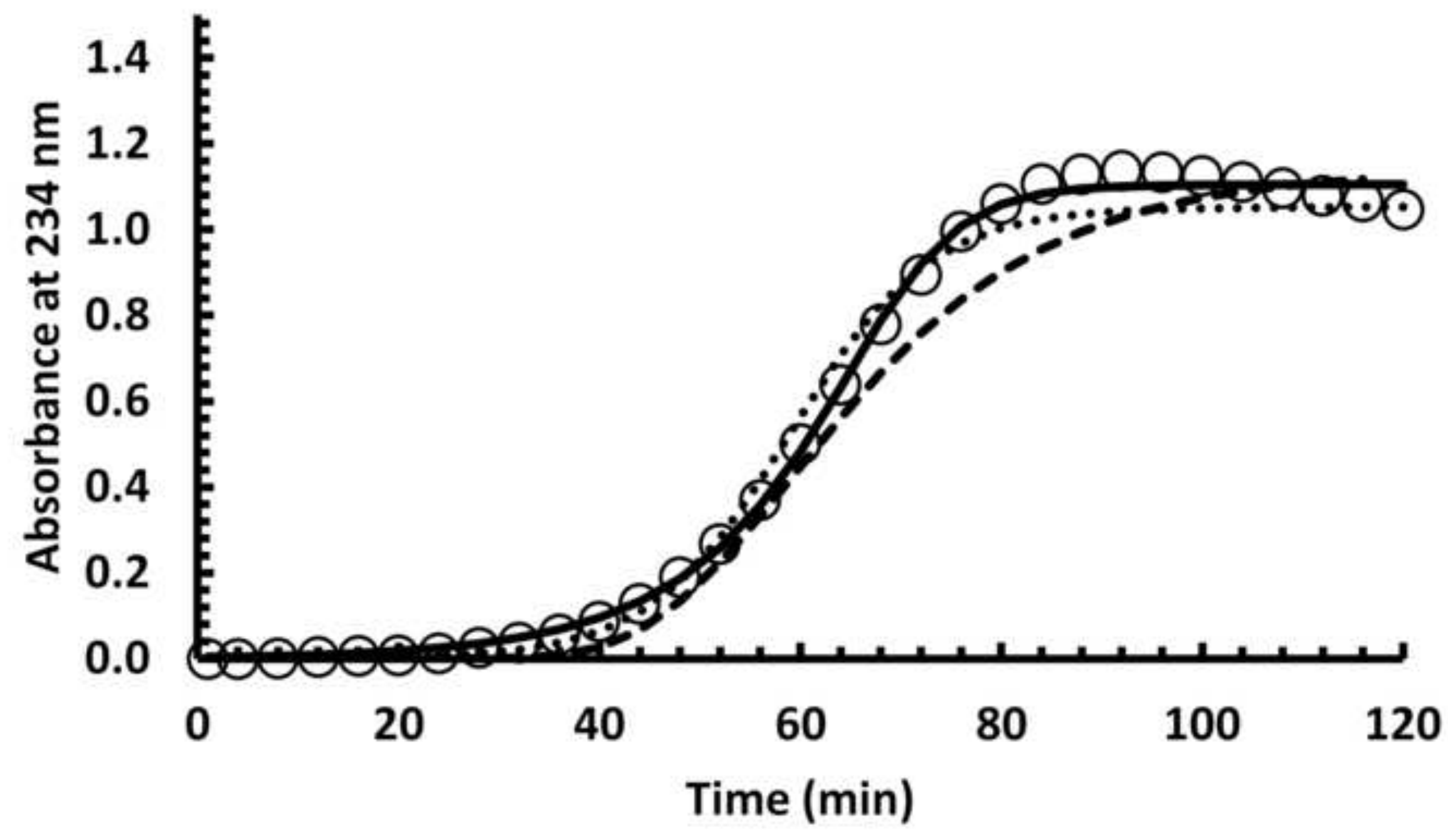




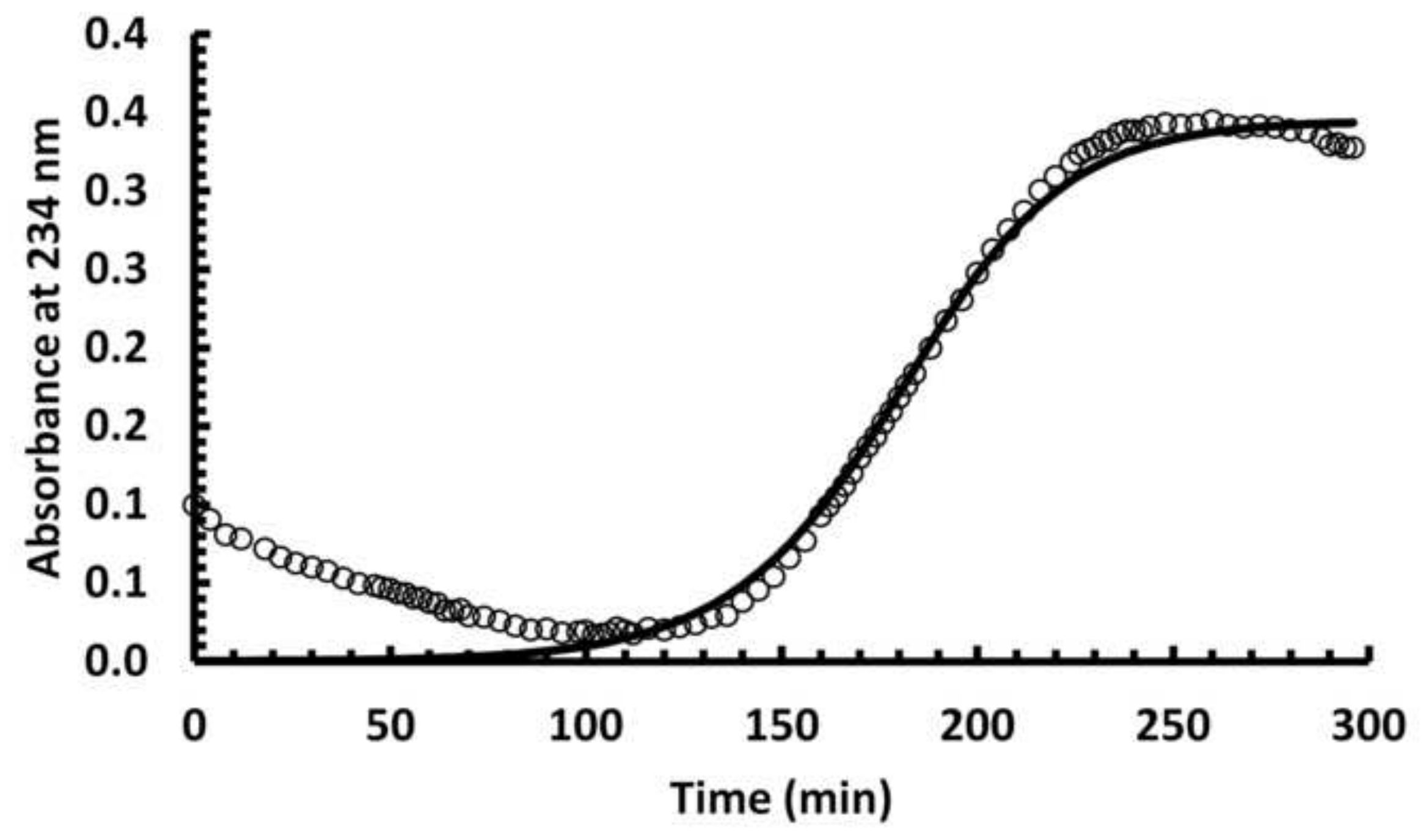




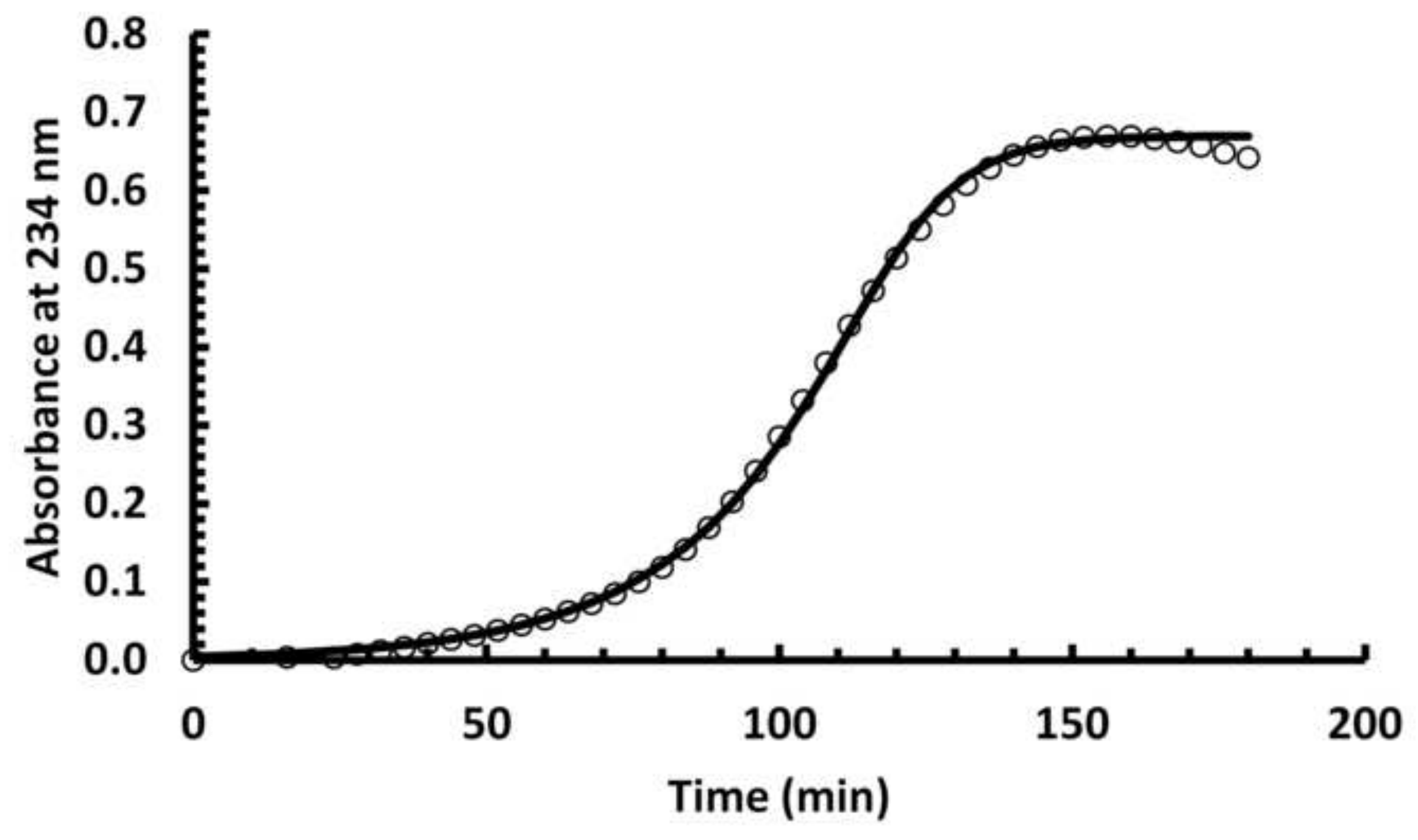




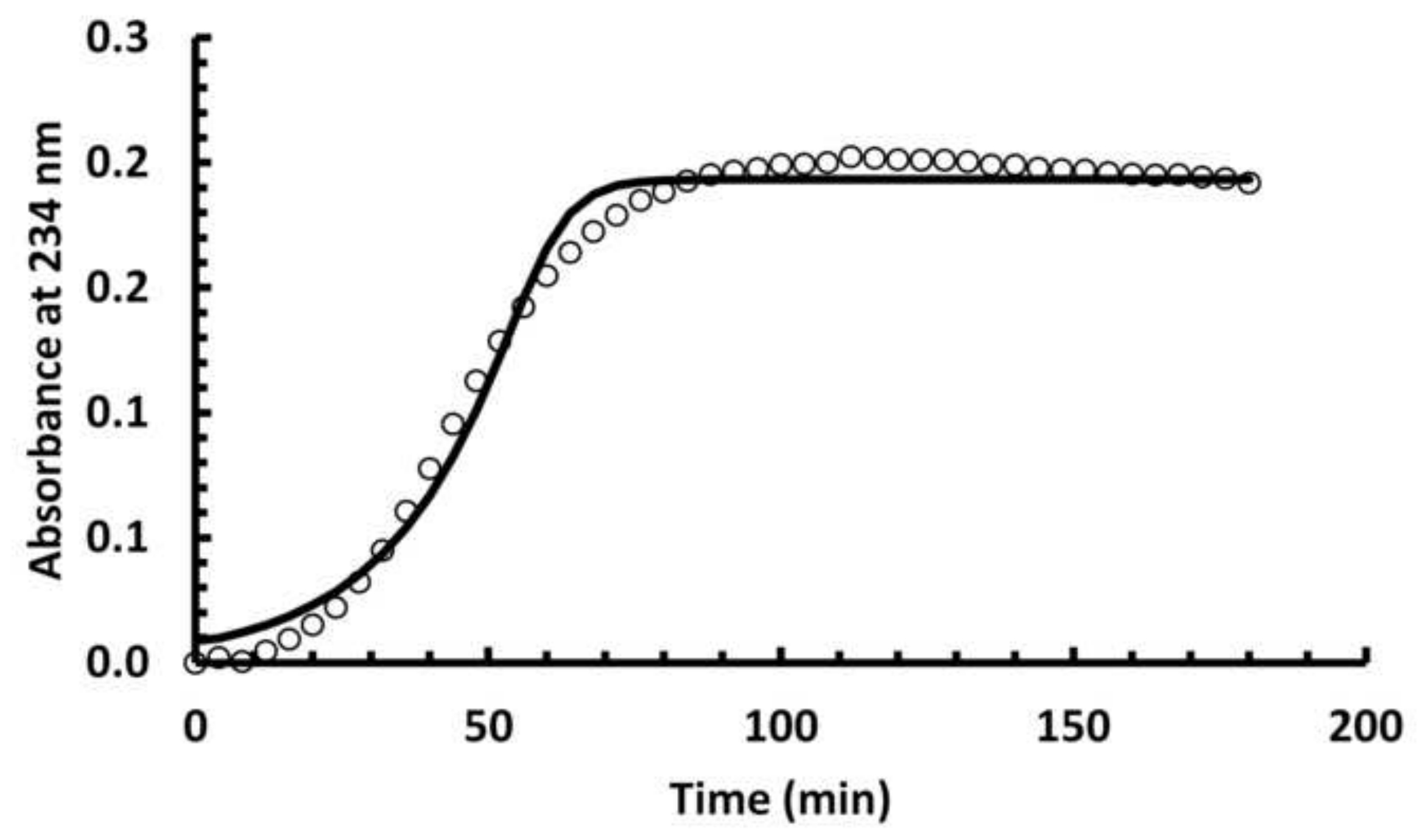


Highlights, McPherson et al. (2012)

- We investigated the application of three sigmoid functions to lipid peroxidation.

- Results demonstrate that the five-parameter Richards' function best described lipid peroxidation.

- This work is applicable not only to lipid peroxidation/antioxidant studies, but to any phenomena observing sigmoid kinetics. 